\title{
AVALIAÇÃO DAS CONDIÇÕES DE ESTOCAGEM DE VACINAS VIVAS, ATENUADAS CONTRA O SARAMPO, EM POSTOS DE VACINAÇÃO CREDENCIADOS E EM CENTROS DE SAUDE DO ESTADO DE SÃO PAULO (BRASIL)*
}

\author{
Inácio França Mendes** \\ Michel Marie Pral** \\ Cosue Miyaki** \\ Neuza Maria Frazatti Gallina** \\ Vera Lucia Petricevich** \\ Francisco Liauw Woe Fang** \\ Hiroko Nakahata Tuchiya** \\ Tuyoshi Ninomya*** \\ Edda de Rizzo**
}

\begin{abstract}
MENDES, I.F, et al. Avaliação das condições de estocagem de vacinas vivas, atenuadas contra o sarampo, em postos de vacinação credenciados e em centros de saúde do Estado de São Paulo (Brasil). Rev. Saúde públ., S. Paulo, $19: 444-9,1985$.
\end{abstract}

RESUMO: Para avaliar as condições de estocagem de vacinas vivas, atenuadas contra o sarampo, da rede de vacinação do Estado de São Paulo (Brasil), foram visitados 71 Postos de Vacinação Credenciados particulares (PVC), assim como $117 \mathrm{Cen-}$ tros de Saúde oficiais (CS), sobre os quais interessava saber a respeito da qualidade da estocagem a frio. Os parâmetros adotados foram: a) temperatura das geladeiras de uso $\left(+2 \mathrm{a}+8^{\circ} \mathrm{C}\right)$ e de estoque $\left(+8^{\circ} \mathrm{C}\right)$; b) validade do produto; c) título das vacinas conservadas nestas geladeiras, avaliado pela inoculação de diluições das amostras de vacinas em células Vero; d) proteção à luz. Dos CS pesquisados, $85,33 \%$ apresentaram geladeiras com temperatura de acordo com a recomendada e $100 \%$ das vacinas neles estocadas com título e validade satisfatórios. Nos PVC foram encontrados, com maior freqüência, lotes de vacina fora do prazo de validade $(14,49 \%)$, com títulos abaixo do mínimo requerido $(3,53 \%)$ e geladeiras de uso e de estoque com temperaturas inadequadas $(33,80 \%)$. Necessário se faz que as condições de estocagem das vacinas contra o sarampo (temperatura e proteção à luz), prevalentes no momento, sejam melhoradas e que as bulas passem a acompanhar o produto a eles entregue, para que os responsáveis pela vacinação obedeçam as recomendações do laboratório produtor com relação às condições de estocagem, validade e administração do imunobiológico, uma vez que a pesquisa revelou que estas não são observadas com o rigor necessário.

UNITERMOS: Sarampo, vacina. Postos de Vacinação Credenciados. Centros de Saúde. Controle de qualidade. Avaliação, estudos.

\section{INTRODUÇÃO}

Para desencadear imunidade efetiva, a vacina viva, atenuada contra o sarampo, deve apresentar concentração de vírus $\geqslant 1.000$ DICT $_{50}$ (doses infectantes de cultura de tecidos $50 \%$ ), a qual é pre- servada pela estocagem em temperaturas de $+2 \mathrm{a}+8^{\circ} \mathrm{C}$ ou de $-20^{\circ} \mathrm{C}^{1,2,3}$.

De 1976 a 1981, o Instituto Butantan titulou todos os lotes de vacina usados nos Centros de Saúde (CS) na vacinação

- Realizado com o auxílio financeiro do FESIMA - Fomento de Educação Sanitária e Imunização em Massa contra Doenças Transmissiveis (Proc. n.'s 289/83, 362/83 e 437/83) e FEDIB - Fundo Especial de Despesas do Instituto Butantan. Apresentado no II Encontro Nacional de Virsicisu, São Lourenço, MG, novembro/1984:

* Da Secão de Cultura de Tecidos e Controle, Serviço de Virologia do Instituto Butantan - Av. Vital Brasil, 1500 - 05504 - São Paulo, SP - Brasil.

*- Da Secretaria de Estado da Saúde de São Paulo - Av. Dr. Amaldo, 351 - $01255-$ São Paulo, SP $\rightarrow$ Brasil. 
MENDES, I.F. et al. Avaliação das condições de estocagem de vacinas vivas, atenuadas contra o sarampo, em postos de vacinação credenciados e em centros de saúde do Estado de São Paulo (Brasil). Rev. Saúde públ., S. Paulo, 19:444-9, 1985.

da população infantil do Estado de São Paulo ${ }^{4}$. Em 1982, realizou levantamento das condições de estocagem dessas vacinas (cadeia de frio) nos CS do município de São Paulo e da Grande São Paulo, visando a informar à rede de vacinação a qualidade das vacinas por ela administradas, no que se referia à preservação de sua potência.

O presente estudo, desenvolvido em 1983-1984, foi embasado na experiência obtida no levantamento de 1982 e apresenta resultados sobre as condições de estocagem vigentes em $117 \mathrm{CS}$ do município de São Paulo, Grande São Paulo, Vale do Paraíba e Sorocaba, assim como em 71 Postos de Vacinação Credenciados (PVC) do Município de São Paulo e da Grande São Paulo. Nele foi dado ênfase à verificação de: a) temperatura das geladeiras em que as vacinas de estoque (vacinas liofilizadas) e de uso (vacinas liofilizadas e/ou reconstituídas para uso imediato) são conservadas; b) proteção das vacinas contra a luz nas referidas geladeiras (liofilizadas e após reconstituição); c) validade das vacinas; d) titulação das amostras de vacinas coletadas em CS e PVC para comprovação da presença ou não do título mínimo requerido pela OMS ${ }^{2}$.

\section{MATERIAL E METODOS}

Obtenção de dados - Os dados sobre a estocagem e utilização de vacinas contra o sarampo nos CS e PVC foram obtidos através de entrevistas com os funcionários responsáveis pela sua estocagem e administração, obedecendo a critérios pré-estabelecidos, tanto de contato pessoal, como de execução de trabalho. Os dados foram anotados em formulários padronizados.

Temperatura das geladeiras de uso e de estoque - A temperatura das geladeiras foi aferida com termômetro cali- brado da equipe de inspeção do Instituto Butantan e comparada, quando possível, com a do termômetro existente nas geladeiras dos CS ou dos PVC. O termômetro referência da equipe era colocado junto às vacinas, nas geladeiras de uso e de estoque, lá permanecendo por um período de $10 \mathrm{~min}$, após os quais a temperatura era registrada no formulário.

Proteção contra a luz - Como o vírus do sarampo é sensível à luz, com base nos mapas de vacinação diária existentes em cada unidade, foi calculado o tempo de exposição das vacinas, tanto à luz da lâmpada como à luz do ambiente.

Validade das vacinas - Foi verificada individualmente a validade das vacinas coletadas das geladeiras de uso, para teste. Quando nelas não havia vacinas, foram verificadas as da geladeira de estoque.

Coleta de amostras - Amostras foram colhidas dos lotes de vacina estocadas há mais tempo, em uso no momento da inspeção, colocadas em sacos plásticos, identificadas e imediatamente acondicionadas em caixa térmica, cuja temperatura foi mantida a cerca de $-15^{\circ} \mathrm{C}$, até chegarem ao laboratorio.

Transporte da vacina até o Instituto Butantan - Foi feito em caixa térmica, provida de tampa hermética, contendo gelo seco suficiente para manter a temperatura a cerca de $-15^{\circ} \mathrm{C}$, durante a jornada diária de trabalho.

Titulação das amostras coletadas Ao chegarem ao laboratório, as amostras foram estocadas a $-20^{\circ} \mathrm{C}$, até serem submetidas a teste de potência ${ }^{4,5}$.

Nos cassos de não verificação dos parâmetros levantados, o fato deveu-se a geladeiras estarem com avarias ou em rotina de manutenção e/ou à inexistência de amostras de vacinas nos postos inspecionados. 
MENDES, I.F, et al. Avaliação das condições de estocagem de vacinas vivas, atenuadas contra o sarampo, em postos de vacinação credenciados e em centros de saúde do Estado de São Paulo (Brasil). Rev. Saúde públ., S. Paulo, 19:444-9, 1985.

\section{RESULTADOS E DISCUSSÃO}

A Tabela 1 apresenta resultados do levantamento realizado em $30 \mathrm{CS}$ da Região de Sorocaba e em 24 CS da Região do Vale do Paraíba.

A temperatura das geladeiras em que imunobiológicos para uso são mantidos, incluindo a vacina contra o sarampo, deve estar na faixa de $+2 \mathrm{a}+8^{\circ} \mathrm{C}$, para preservar suas características. Dos 24 CS inspecionados no Vale do Paraíba, $79,17 \%$ apresentaram suas geladeiras de uso dentro desse limite, enquanto que, em Sorocaba, $75 \%$ o fizeram. Quanto à temperatura das geladeiras de estoque, as duas regiões apresentaram níveis praticamente comparáveis, isto é,
$100 \%$ para o Vale do Paraíba e $96,55 \%$ para Sorocaba. Os menores percentuais relacionados com a temperatura das geladeiras de uso parecem ser consequiência do elevado número de vezes que esses equipamentos são abertos durante a jornada de trabalho dos CS.

Nas duas regiões, as condições de proteção das vacinas contra a luz foram insatisfatórias. Entretanto, em ambas, $100 \%$ das vacinas existentes tanto nas geladeiras de uso, como nas de estoque, estavam dentro do prazo de validade e com título (potência) requerido.

Os dados obtidos na inspeção realizada em 63 CS e em 71 PVC do município de São Paulo e da Grande São Paulo

\section{TABELA 1}

Dados percentuais obtidos na avaliação das condições de estocagem da vacina viva, atenuada, contra o sarampo nos Centros de Saúde de duas regiōes do Estado de São Paulo

\begin{tabular}{|c|c|c|c|c|c|c|c|c|}
\hline \multirow{3}{*}{$\begin{array}{c}\text { Condições } \\
\text { de } \\
\text { Estocagem }\end{array}$} & \multicolumn{8}{|c|}{$\begin{array}{l}\text { Região de Sorocaba } \\
\mathrm{N}^{\circ} \text { inspecionado: } 30\end{array}$} \\
\hline & \multicolumn{2}{|c|}{$\begin{array}{l}\text { Temperatura da } \\
\text { Geladeira }\end{array}$} & \multicolumn{2}{|c|}{ Proteção à luz } & \multicolumn{2}{|c|}{ Validade } & \multicolumn{2}{|c|}{ Título } \\
\hline & Uso & Estoque & Uso & Estoque & Uso & Estoque & Uso & Estoque \\
\hline De acordo & $\begin{array}{c}21 \\
75,00 \\
\end{array}$ & $\begin{array}{r}28 \\
96,55 \\
\end{array}$ & $\begin{array}{c}01 \\
3,70 \\
\end{array}$ & $\begin{array}{c}14 \\
46,67 \\
\end{array}$ & $\begin{array}{c}10 \\
100,0 \\
\end{array}$ & $\begin{array}{c}25 \\
100,0 \\
\end{array}$ & $\begin{array}{c}10 \\
100,0 \\
\end{array}$ & $\begin{array}{c}26 \\
100,0 \\
\end{array}$ \\
\hline Em desacordo & $\begin{array}{r}07 \\
25,00 \\
\end{array}$ & $\begin{array}{l}01 \\
3,45\end{array}$ & $\begin{array}{r}26 \\
96,30 \\
\end{array}$ & $\begin{array}{c}16 \\
53,33 \\
\end{array}$ & 0 & 0 & 0 & 0 \\
\hline Não verificadas & 02 & 01 & 03 & 0 & 1 & 05 & 20 & 05 \\
\hline \multirow{3}{*}{$\begin{array}{l}\text { Condições } \\
\text { de } \\
\text { Estocagem }\end{array}$} & \multicolumn{8}{|c|}{$\begin{array}{l}\text { Região do Vale do Paraíba } \\
\text { NQ inspecionado: } 24\end{array}$} \\
\hline & \multicolumn{2}{|c|}{$\begin{array}{c}\text { Temperatura da } \\
\text { Geladeira }\end{array}$} & \multicolumn{2}{|c|}{ Proteção à luz } & \multicolumn{2}{|c|}{ Validade } & \multicolumn{2}{|c|}{ Título } \\
\hline & Uso & Estoque & Uso & Estoque & Uso & Estoque & Uso & Estoque \\
\hline De acordo & $\begin{array}{c}19 \\
79,17 \\
\end{array}$ & $\begin{array}{r}24 \\
100,0 \\
\end{array}$ & $\begin{array}{c}02 \\
8,33 \\
\end{array}$ & $\begin{array}{l}01 \\
4,17 \\
\end{array}$ & $\begin{array}{c}04 \\
100,0 \\
\end{array}$ & $\begin{array}{c}22 \\
100,0 \\
\end{array}$ & $\begin{array}{c}04 \\
100,0 \\
\end{array}$ & $\begin{array}{c}22 \\
100,0 \\
\end{array}$ \\
\hline Em desacordo & $\begin{array}{c}05 \\
20,83 \\
\end{array}$ & 0 & $\begin{array}{r}22 \\
91,67 \\
\end{array}$ & $\begin{array}{r}23 \\
95,83 \\
\end{array}$ & 0 & 0 & 0 & 0 \\
\hline Não verificadas & 0 & 0 & 0 & 0 & 20 & 02 & 0 & $\mathbf{0}$ \\
\hline
\end{tabular}


MENDES, I.F. et al. Avaliação das condiçōes de estocagem de vacinas vivas, atenuadas contra o sarampo, em postos de vacinação credenciados e em centros de saúde do Estado de São Paulo (Brasil). Rev. Saúde públ., S. Paulo, 19:444-9, 1985.

constam da Tabela 2. Nos CS existem duas geladeiras tipo doméstico, uma para produtos em uso e outra para seu estoque. Já os PVC apresentam uma só geladeira, tanto para produtos de uso imediato como para estoque, equipamentos esses que são geralmente do tipo "console", comuns nos consultórios médicos. Poí esse motivo, na Tabela em questão não são apresentados dados referentes às geladeiras de estoque. Observouse que nos CS inspecionados, $80,33 \%$ das geladeiras de uso e $80,95 \%$ das de estoque apresentaram temperatura de acordo com a recomendada $(+2$ a $+8^{\circ} \mathrm{C}$ ), enquanto que nos PVC, $66,20 \%$ das geladeiras conservavam o produto sob temperatura adequada. Com relação à proteção da vacina contra a luz, tanto os CS quanto os PVC apresentaram resultados pouco satisfatórios. Nos CS, $100 \%$ das vacinas das geladeiras de uso e de estoque estavam dentro do prazo de validade e com a potência requerida. O mesmo não ocorreu nos PVC, nos quais, mesmo pertencendo à mesma área geográfica dos CS, $14,49 \%$ das vacinas estavam fora do prazo de validade, e $3,53 \%$ com título abaixo do mínimo requerido, que é de $1.000 \mathrm{DICT}_{30} /$ dose.

TABELA 2

Dados percentuais obtidos na avaliação das condições de estocagem da vacina viva, atenuada. contra o sarampo nos Centros de Saúde e Postos de Vacinação Credenciados das regiōes do Município de São Paulo e Grande São Paulo

\begin{tabular}{|c|c|c|c|c|c|c|c|c|}
\hline \multirow{3}{*}{$\begin{array}{l}\text { Condições } \\
\text { de } \\
\text { Estocagem }\end{array}$} & \multicolumn{8}{|c|}{$\begin{array}{l}\text { Centros de Saúde } \\
\text { No inspecionado: } 63\end{array}$} \\
\hline & \multicolumn{2}{|c|}{$\begin{array}{l}\text { Temperatura da } \\
\text { Geladeira }\end{array}$} & \multicolumn{2}{|c|}{ Proteção à luz } & \multicolumn{2}{|c|}{ Validade } & \multicolumn{2}{|c|}{ Título } \\
\hline & Uso & Estoque & Uso & Estoque & Uso & Estoque & Uso & Estoque \\
\hline De acordo & $\begin{array}{c}49 \\
80,33\end{array}$ & $\begin{array}{c}51 \\
80,95\end{array}$ & $\begin{array}{c}03 \\
4,84\end{array}$ & $\begin{array}{c}20 \\
33,90\end{array}$ & $\begin{array}{c}29 \\
100,0\end{array}$ & $\begin{array}{c}34 \\
100,0\end{array}$ & $\begin{array}{c}29 \\
100,0\end{array}$ & $\begin{array}{c}34 \\
100,0\end{array}$ \\
\hline Em desacordo & $\begin{array}{c}12 \\
19,67 \\
\end{array}$ & $\begin{array}{c}12 \\
19,05 \\
\end{array}$ & $\begin{array}{c}59 \\
95,16 \\
\end{array}$ & $\begin{array}{c}39 \\
66,10 \\
\end{array}$ & 0 & 0 & 0 & 0 \\
\hline Não verificadas & 02 & 0 & 01 & 04 & 34 & 29 & 34 & 29 \\
\hline \multirow{3}{*}{$\begin{array}{l}\text { Condições } \\
\text { de } \\
\text { Estocagem }\end{array}$} & \multicolumn{8}{|c|}{$\begin{array}{c}\text { Postos de Vacinação Credenciados } \\
\text { No inspecionado: } 71\end{array}$} \\
\hline & \multicolumn{2}{|c|}{$\begin{array}{l}\text { Temperatura da } \\
\text { Geladeira }\end{array}$} & \multicolumn{2}{|c|}{ Proteção à luz } & \multicolumn{2}{|c|}{ Validade } & \multicolumn{2}{|c|}{ Título } \\
\hline & Uso & Estoque & Uso & Estoque & Uso & Estoque & Uso & Estoque \\
\hline De acordo & \multicolumn{2}{|c|}{47} & \multicolumn{2}{|r|}{17} & & $\begin{array}{c}59 \\
85,51\end{array}$ & \multicolumn{2}{|c|}{82} \\
\hline Em desacordo & \multicolumn{2}{|c|}{$\begin{array}{c}24 \\
33,80 \\
\end{array}$} & \multicolumn{2}{|r|}{$\begin{array}{c}54 \\
76,06\end{array}$} & & $\begin{array}{c}10 \\
14,49 \\
\end{array}$ & \multicolumn{2}{|c|}{$\begin{array}{c}03 \\
3,53\end{array}$} \\
\hline Não verificadas & \multicolumn{2}{|r|}{0} & \multicolumn{2}{|r|}{0} & & 02 & \multicolumn{2}{|c|}{04} \\
\hline
\end{tabular}


MENDES, I.F. et al. Avaliação das condições de estocagem de vacinas vivas, atenuadas contra o sarampo, em postos de vacinação credenciados e em centros de saúde do Estado de São Paulo (Brasil). Rev. Saúde públ., S. Paulo, 19:444-9, 1985.

A comparação dos resultados obtidos na avaliação das condições de estocagem das vacinas: em geladeiras de uso e de estoque de CS e de PVC encontra-se na Tabela 3. Do total dos 117 CS do Estado de São Paulo pesquisados, apesar de $14,67 \%$ e de $83,06 \%$ das vacinas não estarem convenientemente estocadas com relação à temperatura e à luz, respectivamente, dentre as amostras de vacinas neles coletadas, nenhuma foi encontrada fora do prazo de validade e/ou com o título abaixo do requerido. Com relação aos 71 PVC pesquisados, as percentagens foram mais desfavoráveis, isto é, $33,80 \%$ não estavam na temperatura ideal e $76,06 \%$ não estavam protegidas contra a luz. Nesses locais, foram encontrados imunobiológicos tanto fora de validade, como com título que não obedecia às exigências da Organização Mundial da Saúde.

TABELA 3

Análise comparativa dos resultados da avaliação das condições de estocagem da vacina viva, atenuada, contra o sarampo nos Centros de Saúde (CS) e Postos de Vacinação Credenciados (PVC) do Estado de São Paulo

\begin{tabular}{|c|c|c|c|c|}
\hline \multirow{2}{*}{$\begin{array}{l}\text { Condições } \\
\text { de } \\
\text { Estocagem }\end{array}$} & \multicolumn{2}{|c|}{$117 \mathrm{CS}$} & \multicolumn{2}{|c|}{$71 \mathrm{PVC}$} \\
\hline & $\begin{array}{l}\text { De acordo } \\
\%\end{array}$ & $\begin{array}{c}\text { Em desacordo } \\
\%\end{array}$ & $\begin{array}{c}\text { De acordo } \\
\%\end{array}$ & $\begin{array}{c}\text { Em desacordo } \\
\%\end{array}$ \\
\hline $\begin{array}{l}\text { Temperatura } \\
\text { das } \\
\text { Geladeiras }\end{array}$ & 85,33 & 14,67 & 66,20 & 33,80 \\
\hline $\begin{array}{l}\text { Proteção da } \\
\text { Vacina contra } \\
\text { a luz } \\
\end{array}$ & 16,94 & 83,06 & 23,94 & 76.06 \\
\hline $\begin{array}{c}\text { Validade } \\
\text { das } \\
\text { Vacinas }\end{array}$ & 100 & 0 & 85,51 & 14,49 \\
\hline $\begin{array}{l}\text { Título } \\
\text { das } \\
\text { Vacinas }\end{array}$ & 100 & 0 & 96,47 & 3,53 \\
\hline
\end{tabular}

A pesquisa evidenciou que a estocagem das vacinas vivas, atenuadas, contra o sarampo, ainda apresenta problemas sob certos aspectos, tanto nos CS, como nos PVC do Estado de São Paulo. Necessário se torna, pois, que medidas efetivas sejam adotadas, de modo que as indicações do laboratório produtor do imunobiológico, referentes à sua conservação e uso, sejam plenamente obedecidas.

\section{AGRADECIMENTOS}

A José Luís Rosseto e José Antonio Padula pelo trabalho técnico. 
MENDES, I.F. et al. Avaliação das condiçōes de estocagem de vacinas vivas, atenuadas contra o sarampo, em postos de vacinação credenciados e em centros de saúde do Estado de São Paulo (Brasil). Rev. Saúde públ., S. Paulo, 19:444-9, 1985.

MENDES, I.F. et al. [An assessment of the storage conditions of live, attenuated vaccines against measles, in authorized vaccination centers and in health services in $S$. Paulo State (Brazil) ]. Rev. Saúde públ., S. Paulo, $19: 444-9,1985$.

ABSTRACT: In the State of S. Paulo, Brazil, health centers sponsored by the State, as well as private health services, located in throughout large districts, are in charge of the vaccination against the various diseases affecting children, including measles. In the present study three of the above mentioned districts, covering 385 State Health Centers (SHC) and 200 Private Health Services (PHS) were surveyed. From these totals 117 SHC and 71 PHS were chosen for the evaluation of: a) quality of the existing cold chain for measles vaccine storage (temperatura); b) vaccine titer; c) validity; d) protection from light. Of the SHC inspected, $85.33 \%$ kept vaccines at the recommended temperature, $100 \%$ presented vaccines with titers above the minimum required and within expiration date. Deficiency relating to protection from light, was found in $83.06 \%$. Inspection of the PHS revealed that whereas $66.20 \%$ kept vaccine within the right temperature range, $76.06 \%$ did not protect them against light, only $96.47 \%$ had vaccines with potency above the level required and only $85.51 \%$, observed the expiration date. The study led to the conclusion that in the State of $\mathrm{S}$. Paulo, measles vaccines are better stored in the State Health Centers than in the Private Health Services surveyed indicating that the slight preference that parents show towards having their children vaccinated in PHS is not justified. It was suggested that some conditions of measles vaccine storage montly, temperature and protection from light should be improved and that instruction leaflets must always accompany immunobiological materials distributed to all health centers, to be consulted when doubts about the proper storage and administration of these vaccines arise.

UNITERMS: Measles, vaccine. Community Health Services. Private Health Services. Quality control. Evaluating studies.

\section{REFERENCIAS BIBLIOGRÁFICAS}

1. BIOLOGICAL products: regulations: title 42. Bethesda, Md, Public Health Service, 1969. Part 73, p. 54-8.

2. CODE of federal regulations. Bethesda, Mr, Public Health Service, 1982. (Food and Drugs, 21, parts 600-1.299).

3. MINISTERIO DA SAÚdE. Programa Nacional de Imunizações. Manual de vacinação. Brasília, 1983.
4. PRAL, M.M. et al. Potency control of live, attenuated vaccines against measles used in children vaccinations in the State of São Paulo. Rev. Inst. Med. trop. S. Paulo, 24: 1-5, 1982.

5. REED, L.S. \& MUENCH, H. A simple method of stimating fifty per cent end points. Amer. J. Hyg., 27:493-7, 1983.

Recebido para publicação em 03/04/1985 Reapresentado em 17/07/1985

A provado para putilicação em 09/08/1985 


\section{E R R A T A}

REVISTA DE SAUDE PUBLICA, 19(5), 1985

p. 444, Resumo - 6? linha

Onde se lê: ...uso $\left(+2 \mathrm{a}+8^{\circ} \mathrm{C}\right)$ e de estoque $\left(+8^{\circ} \mathrm{C}\right)$; b) validade... Leia-se: ...uso $\left(+2 \mathrm{a}+8^{\circ} \mathrm{C}\right)$ e de estoque $\left.\left(\leqslant+8^{\circ} \mathrm{C}\right) ; \mathrm{b}\right)$ validade...

p. 446, Tabela 1 - na última linha, na 5 a coluna, o valor é 20

p. 449, Abstract - 18? linha

Onde se lê: ... of measles vaccine storage montly, temperature...

Leia-se: $\ldots$ of measles vaccine storage, mostly temperature... 
O arquivo disponível sofreu correções conforme ERRATA publicada no Volume 20 Número 1 da revista. 